\title{
I-gel as a first-line airway device in the emergency room for patients with out-of-hospital cardiac arrest
}

\author{
DONG KEON LEE ${ }^{1}$, DONG HYUCK SHIN², SEUNG MIN PARK ${ }^{1}$, YONG HWAN KIM ${ }^{3}$, SANG O PARK, YOUNG HWAN LEE \\ 1 Department of Emergency Medicine, Seoul National University Bundang Hospital, Republic of Korea \\ 2 Department of Emergency Medicine, Kangbuk Samsung Hospital, Sungkyunkwan University School of Medicine, Seoul, Republic of Korea \\ 3 Department of Emergency Medicine, Samsung Changwon Hospital, Sungkyunkwan University School of Medicine, Changwon, Republic of Korea \\ 4 Department of Emergency Medicine, Konkuk University Medical Centre, Konkuk University School of Medicine, Seoul, Republic of Korea \\ 5 Department of Emergency Medicine, College of Medicine, Soonchunhyang University, Bucheon, Gyeonggi-do, Republic of Korea
}

Corresponding author:

Young Hwan Lee

Department of Emergency Medicine

College of Medicine

Soonchunhyang University

14584, 170, Jomaru-ro, Bucheon-si

Gyeonggi-do, Republic of Korea

Phone: +82-10-4047-4270

Fax: +0504-428-0908

E-mail:zerohwani@gmail.com

\section{ABSTRACT}

Aim. The optimal method for advanced airway management during cardiac arrest remains controversial. Most patients with out-of-hospital cardiac arrest (OHCA) in Korea are managed with a bag-valve mask by paramedics, while physicians perform advanced airway management in emergency departments (ED). Endotracheal intubation (ETI) has a risk of failure at the first attempt. By contrast, I-gel, a supraglottic airway device, is easier to insert than an endotracheal tube and shows a higher first-attempt success rate than ETI in outof-hospital settings by paramedics in the United States. We reviewed the use of ETI and I-gel by ED physicians to assess the first attempt success rate in a hospital setting. Methods. We conducted a retrospective chart review of patients with non-traumatic OHCA who were managed with either ETI using a Macintosh laryngoscope, or I-gel in the ED of Korean hospital from January 2012 to January 2014.

Results. Of 322 adult patients with nontraumatic OHCA, 160 received I-gel and 162 received ETI. The first-attempt success rate was higher in the I-gel group (96.9\%) than in the ETI group (84.6\%, p < 0.001). The time from arrival to obtaining advanced airway management was shorter in the I-gel group than in the ETI group.

Conclusions. I-gel showed a better firstattempt success rate and shorter insertion time compared with ETI when performed by physicians in a hospital setting.
Key words: airway management, laryngeal mask, out-of-hospital cardiac arrest, resuscitation

\section{INTRODUCTION}

In patients with cardiac arrest, high-quality chest compression and effective ventilation are key components of successful resuscitation. However, the optimal method for advanced airway management during cardiac arrest remains controversial. (1)

In the United States, paramedics perform endotracheal intubation (ETI) or supraglottic airway (SGA) device insertion in patients with out-of-hospital cardiac arrest (OHCA) for early airway management. (2) In Korea, most emergency medical service (EMS) paramedics use a bag-valve mask (BVM) to manage patients with OHCA. $(3,4)$ In this situation, the emergency department (ED) physician has to perform advanced airway management when the patient arrives at the ED. Most ED physicians consider ETI as a first choice for inserting a first-line airway device. $(5,6)$ However, according to the 2010 American Heart Association (AHA) guidelines, ETI has a possibility of failure including unrecognized esophageal intubation or delayed insertion of an airway device. (7) The failure rate of ETI on the first attempt by ED physician in the ED or an in-hospital setting varies from $20 \%$ to $32 \%$. (5) Continual and multiple intubation attempts are associated with respiratory problems, (8) and a failed initial intubation attempt is a risk fac- tor for decreased effectiveness of advanced cardiac life support (ACLS) in patients with OHCA. (5)

By contrast, a laryngeal mask airway (LMA) is a more successful adjustment than an endotracheal tube, (9) and many studies have compared the success rate of LMA with that of ETI. $(10,11)$ Moreover, I-gel has been introduced as a second-generation SGA device. I-gel is an emerging SGA device with a gel-like, non-inflatable cuff designed to create a non-inflatable anatomical seal around the pharyngeal, laryngeal and perilaryngeal structures. (12) Several studies have reported that I-gel shows a higher success rate compared with ETI and other SGA devices when used in an out-of-hospital setting by paramedics. $(12,13)$ However, there has been no study to investigate the utility of I-gel when used by physicians in an in-hospital setting. Moreover, since most patients with OHCA are very urgent, there could be no guardians at the scene. For these reasons, authors designed a chart review study for verifying the possibility of I-gel as a first-line airway device when used by ED physicians for patients with OHCA in ED. We set the primary objective of this study as the first attempt success rate of each device.

\section{MATERIAL AND METHODS}

Study design

This is a retrospective chart review study. Patients included were those with OHCA 
Table 1. Characteristics of patient participants according to the type of advanced airway management device used

\begin{tabular}{lll}
\hline & $\begin{array}{l}\text { Endotracheal tube } \\
(\mathbf{n}=\mathbf{1 6 2})\end{array}$ & $\begin{array}{l}\text { I-gel } \\
(\mathbf{n}=\mathbf{1 6 0})\end{array}$ \\
\hline Age, median (range), years & $66.15 \pm 16.3$ & $65.68 \pm 16.0$ \\
\hline Male, n (\%) & $96(59.3)$ & $109(68.1)$ \\
\hline Cardiac origin, n (\%) & $55(34.0)$ & $53(33.1)$ \\
\hline Witnessed by laypersons, n (\%) & $90(55.6)$ & $84(52.5)$ \\
\hline Bystander CPR, n (\%) & $64(39.5)$ & $50(31.3)$ \\
\hline Initial shockable rhythm, n (\%) & $35(21.6)$ & $41(25.6)$ \\
\hline AED by EMS, n (\%) & $32(19.8)$ & $33(32.3)$ \\
\hline Call to CPR by EMS, min & $10.17 \pm 5.61$ & $10.01 \pm 6.18$ \\
\hline CPR by EMS to hospital arrival, min & $17.12 \pm 6.489$ & $17.06 \pm 6.661$ \\
\hline Therapeutic hypothermia & $11(6.8)$ & $10(6.3)$ \\
\hline
\end{tabular}

AED, automated external defibrillator, CPR, cardiopulmonary resuscitation; EMS, emergency medical service.

Table 2. Success rate and time according to the type of advanced airway management device used

\begin{tabular}{llll}
\hline & $\begin{array}{l}\text { Endotracheal tube } \\
(\mathrm{n}=162)\end{array}$ & $\begin{array}{l}\text { I-gel } \\
(\mathrm{n}=160)\end{array}$ & p-value \\
\hline Success rate at first attempt $(\%)$ & $137(84.6)$ & $155(96.9)$ & $<0.001^{*}$ \\
\hline Arrival to advanced airway, $\min ( \pm \mathrm{SD})$ & $2.67( \pm$ & $0.9( \pm$ & $<0.001^{\star}$ \\
\hline Success rate within 1 min, $\mathrm{n}(\%)$ & $7(5.1 \%)$ & $132(85.2 \%)$ & $<0.001^{\star}$ \\
\hline
\end{tabular}

The independent two-sample t-test for continuous variables and a chi-square test for categorical variables were performed.

${ }^{*}$ statistically significant

Table 3. Associations between advanced airway management strategy (I-gel vs endotracheal intubation) and out-of-hospital cardiac arrest outcomes

\begin{tabular}{lllll}
\hline & $\begin{array}{l}\text { Endotracheal tube } \\
(\mathrm{n}=162)\end{array}$ & $\begin{array}{l}\text { I-gel } \\
(\mathrm{n}=160)\end{array}$ & OR (95\%CI) & p-value \\
\hline Sustained ROSC, $\mathrm{n}(\%)$ & $62(38.3)$ & $64(40.0)$ & $1.08(0.69-1.68)$ & 0.751 \\
\hline $\begin{array}{l}\text { Survival to hospital admission, } \\
\mathrm{n}(\%)\end{array}$ & $33(20.4)$ & $31(19.4)$ & $0.94(0.54-1.62)$ & 0.823 \\
\hline $\begin{array}{l}\text { Survival to hospital discharge, } \\
\mathrm{n}(\%)\end{array}$ & $11(6.8)$ & $12(7.5)$ & $1.11(0.48-2.60)$ & 0.363 \\
$\begin{array}{l}\text { Favorable neurologic outcomea, 6(3.7) } \\
\mathrm{n}(\%)\end{array}$ & $6(3.8)$ & $1.22(0.3-4.07)$ & 0.983 \\
\hline
\end{tabular}

Comparisons between I-gel and endotracheal intubation were performed by a chi-square test.

ROSC, return of spontaneous circulation.

aCerebral performance category (CPC) scale 1 or 2 . managed either by I-gel (Intersurgical Ltd, Wokingham, Berkshire, UK) or with ETI at the hospital emergency centre between January 2012 and January 2014. The authors assert that all procedures contributing to this work comply with the ethical standards of the relevant national and institutional committees on human experimentation and with the Helsinki Declaration of 1975, as revised in 2008. The study was approved by our Institutional Review Board (HUSHHIRB, 2016-I 075).

\section{Settings}

The patients in this study were admitted to the tertiary referral centre covering a local population of approximately 600,000 . We have used I-gel since January 2012. We used only I-gel on patients with OHCA who arrived at our ED from January 2012 to January 2013 and only ETI from January 2013 to January 2014.

The EMS system in Korea is a single-tier, basic life-support ambulance service. In Korea, most patients with OHCA are transported with BVM ventilation or an LMA inserted by paramedics. Every OHCA patient who arrived at our ED was managed by a resuscitation team consisting of emergency medicine physicians, emergency medicine residents, emergency medical technicians (EMT) and nurses. The ACLS protocol of the resuscitation team was according to the 2010 AHA guidelines. ETI using a Macintosh laryngoscope or I-gel insertion was performed immediately when the patient arrived in the ED. The procedures were performed by an emergency medicine physician or a 3rd - or 4th - grade emergency medicine senior resident with a valid ACLS certification. All physicians involved had experienced at least 60 cases of ETI in the ED before this study. (14)

\section{Data acquisition}

Chart review was performed by 2 particularly trained chart reviewers. They were blinded to the hypothesis and outcome of this study and performed chart review individually. The chart consists of our own recording form created according to Utstein style and includes demographic information, defibrillator-data, a flow chart of resuscitation and discharge information of every resuscitated patient in ED. Every flow of resuscitation for the patient with OHCA was recorded by the physician, EMT and nurse. This charting system had been conducted on every cardiac arrest patient before this study was designed. When 


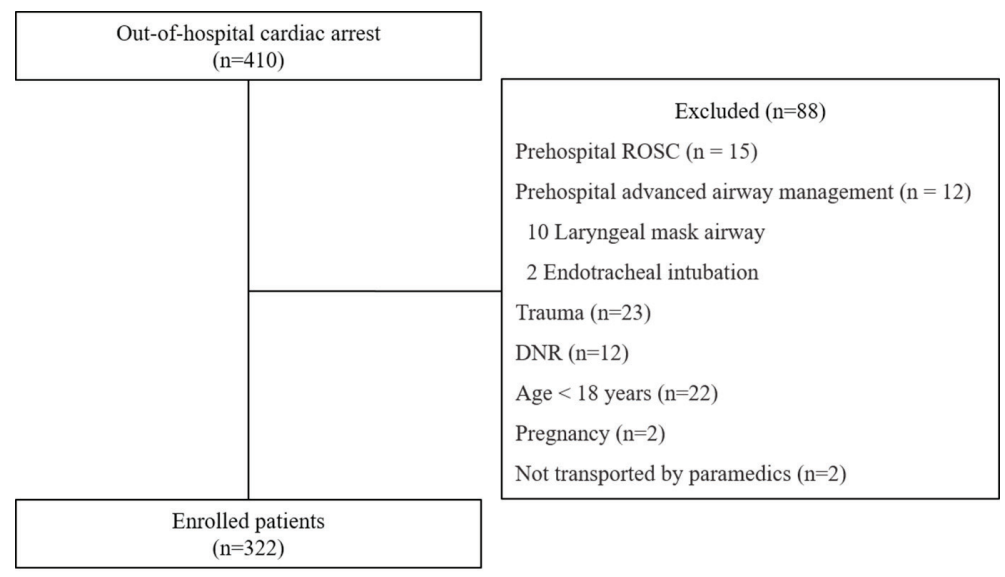

Figure 1. Out-of-hospital cardiac arrest patients between January 2012 and January 2014 $D N R$, did not resuscitate; ROSC, return of spontaneous circulation.

cardiopulmonary resuscitation (CPR) was finished, the chart reviewer filled in our own recording form of the resuscitated patients by reviewing the patient charts, nursing records, and records by EMT. There was no missing or incomplete data during this study.

\section{Study inclusion/exclusion}

The patients included in the study were those with an OHCA and aged $\geq 18$, while patients who had any advanced airway management (LMA or ETI) before they arrived at the hospital, had a traumatic arrest, were in Do not Resuscitate (DNR) state, were pregnant, were missing documents, or were not transported by paramedics, were excluded from the study.

\section{Study outcomes}

The primary outcome was the first-attempt success rate. Most cases of ETI in this study were confirmed by capnography. To confirm successful placement of airway in the I-gel group, we used the multiple assessments including confirming of chest rising and auscultation of both lung field in each case by case. The secondary outcomes were the time from arrival to inserting the airway device, sustained return of spontaneous circulation (ROSC), survival to hospital admission, survival to hospital discharge and favourable neurologic outcome.

Sustained ROSC was defined as maintaining spontaneous circulation for more than 20 minutes. I-gel was changed to ETI when sustained ROSC was established. Neurologic outcome was assessed based on the Cerebral Performance Category scale (CPC). (15) A favourable neurologic outcome was defined as CPC scale 1 or 2.

\section{Statistical analysis}

Statistical analyses were performed using SPSS software for Windows (V.18.0 K, SPSS, Chicago, IL, USA). Categorical data are expressed as number and frequency. Continuous data are presented as mean with standard deviation (SD), or median with interquartile range (IQR) after assessments for normality using the ShapiroWilk test.

Differences between the two groups were tested using an independent two-sample ttest or a Mann-Whitney U test for continuous variables, while a chi-square test, or Fisher's exact test was used for categorical variables. A p $<0.05$ was considered significant. We used SPSS software (version 18.0; SPSS Inc, Chicago, IL, USA) for our analyses.

\section{RESULTS}

Of the 410 patients with OHCA included in our study, $15(3.7 \%)$ patients who had prehospital ROSC, and 12 (2.9\%) patients who had advanced airway management before arrival at the ED (LMA was 10, and ETI was 2), 23 (5.6\%) patients with a traumatic arrest, $12(2.9 \%)$ patients in a DNR state, $22(5.4 \%)$ patients younger than 18 , $2(0.5 \%)$ pregnant patients, and $2(0.5 \%)$ who were not transported by paramedics were excluded. Ultimately, 322 patients were included in our study (figure 1).

Table 1 shows the characteristics of eligi- ble participants. There were 162 patients intubated with an endotracheal tube, and 160 patients had I-gel inserted. Two groups were not statistically different in characteristics.

The first-attempt success rate was higher in the I-gel group (96.9\%) than in the ETI group $(84.6 \% ; \mathrm{p}<0.001)$. In the second attempt, every ETI group patient was successfully intubated, but I-gel insertion was successful in 4 out of 5 I-gel group patients. The failed I-gel group patient was intubated with a video laryngoscope. The time from arrival to obtaining advanced airway management (I-gel, $0.9 \pm 1.52 \mathrm{~min}$; ETI, $2.67 \pm 1.70 \mathrm{~min} ; \mathrm{p}<0.001)$ and success rate within 1 minute (I-gel, 85.2\%; ETI, 5.1\%; p $<0.001$ ), was more favorable for the I-gel group (table 2). We used the Bonferroni correction for these variables and the I-gel group showed a more favorable outcome than ETI group after Bonferroni correction. The associations between advanced airway management and patient outcomes were not statistically different for sustained ROSC (I-gel, 40\%; ETI, 38.3\%; p = 0.751), survival to hospital admission (I-gel, 19.4\%; ETI, $20.4 \%$; $\mathrm{p}=0.823$ ), survival to hospital discharge (I-gel, 7.5\%; ETI, 6.8\%; $\mathrm{p}=0.363$ ), or favorable neurologic outcome (I-gel, 3.7\%; ETI, 3.8\%; p = 0.983) (table 3). Sustained ROSC was achieved in $64 / 160$ (40\%) patients in the I-gel group. Two patients did not require mechanical ventilation and two patients expired before application of mechanical ventilation due to re-arrest. Sixty patients required definitive airway management (endotracheal intubation) for mechanical ventilation. The mean time to change from I-gel to ETI was $20 \pm 5 \mathrm{~min}$

\section{DISCUSSION}

Our study shows that I-gel is associated with a higher first-attempt success rate and the time from arrival to advanced airway management was shorter than that in the ETI group.

In particular, I-gel can achieve early advanced airway placement within $1 \mathrm{~min}$ ute from emergency room arrival in over $80 \%$ of arrest patients. Otherwise, only 5 $\%$ of the ETI group patients are successfully intubated within 1 minute. However, other outcomes (sustained ROSC, survival to hospital admission, survival to hospital discharge, favorable neurologic outcome) were not statistically different between the two groups. In our study, ETI was performed by an emergency medicine physician or emergency medicine senior 
resident. The success rate of ETI (84.6\%) was higher than that reported previously (60\%, Kim et al.; $72.9 \%$, Sakles et al. $(5,16)$ Nevertheless, the success rate for I-gel was higher than for ETI in this study.

According to the 2010 AHA guidelines for Cardiopulmonary Resuscitation and Emergency Cardiovascular Care, there are no data to support the routine use of any specific approach to advanced airway management during cardiac arrest. The best method depends on the precise circumstances of the cardiac arrest, local guidelines, and the competence of the rescuer. (7) In Korea, which has a singletiered, basic EMS, BVM (96.9\%) is used more frequently than ETI (1.2\%) and LMA (1.9\%) in patients with OHCA during their transport to a hospital. (3) In this situation, most patients with OHCA had advanced airway management at the $\mathrm{ED}$, and most ED physicians choose ETI for first-line advanced airway management. However, the failure rate of the first in-hospital ED intubation attempt varies from $20 \%$ to $32 \%$. (5) Adequate airway management helps increase the effectiveness of ACLS in patients with cardiac arrest. Prolonged attempts at intubation are harmful if associated with interruption of chest compressions because they compromise coronary and cerebral perfusion. (7) This may be harmful to patients as it compromises the effectiveness of ACLS management. Some studies suggest that ventilation is not essential during the initial $12 \mathrm{~min}$ of resuscitation with untreated arrest intervals of less than 6 min. (17) However, in Korea, the time from call to arrival at ED is approximately 20 minutes. (3) So, when patients with OHCA arrive at ED, not only is a suitable ACLS management important, but also an effective advanced airway management. $(11,18)$ Steve $\mathrm{T}<$ /author $><$ author $>$ Cawley, Rebekah $\mathrm{J}<$ /author $><$ author $>$ Aune, Sverre
$\mathrm{E}<$ /author $><$ author $>$ Angelos, Mark $\mathrm{G}<1$ author $><$ /authors $><$ /contributors $><$ titles $><$ title $>$ Oxygen requirement during cardiopulmonary resuscitation (CPR For this reason, a prompt and suitable placement of the first-line airway device in patients arriving at ED may be an important contributor to patient outcomes in Korea and similar Asian countries. $(3,17)$

I-gel is a newly developed SGA device, has various merits and does not demand a high skill level or major experience. It can be inserted quickly and easily without interrupting chest compressions and used by many rescuers even if they are relatively less skillful. Many studies show that LMA has a higher success rate and is easier to use than ETI. $(10,11,19)$ I-gel is even easier to use than other LMA devices. $(13,19)$ Many papers have compared I-gel with the endotracheal tube. (20-22) However, these studies were performed by paramedics in a pre-hospital setting.

The current study shows that I-gel is easier and quicker in securing the airway than the ETI at the ED for OHCA patients. Since it shows a higher success rate on the first attempt, shorter time from arrival to device placement and higher success rate within 1 minute ( $85.2 \%$ vs. 5.1$)$ were seen. Regarding the clinical outcomes such as a sustained ROSC, survival to hospital admission, survival to hospital discharge and favorable neurologic outcome, there were no significant differences between the two methods in our study. In consideration of these results, I-gel may be a good alternative advanced airway management device in the ED for those arrest patients. However, I-gel has a disadvantage as with other SGA in that it needs to be replaced by endotracheal intubation for those patients who achieve ROSC and require mechanical ventilation.

There are several limitations to our study.
First, this is a retrospective chart review study, therefore not as convincing as a prospective randomized trial. There may be unintended confounders that could not be adequately adjusted for. Second, our study was performed at a single centre and small data only. A larger volume study may follow in the future to clarify the role of I-gel during CPR.

Third, we confirmed successful insertion of I-gel as multiple assessments because of the uncertain efficacy of capnography in the SGA. However, confirming the successful insertion of I-gel is controversial. Fourth, in our study, the mean call to CPR by EMS time is approximately 10 minutes in both groups, and the proportion of patients who received bystander CPR was $39.5 \%$ in ETI group and $31.3 \%$ in the I-gel group. So, many patients who did not receive bystander CPR could have cerebral damage before arriving at emergency room. It could influence our secondary outcomes.

\section{CONCLUSION}

The first attempt at using I-gel had a higher success rate than ETI in an in-hospital setting when used by physicians. I-gel was quicker to insert than endotracheal intubation in time from arrival to establishing advanced airway management. I-gel may be considered as an alternative airway device for physicians in a hospital setting.

\section{ACKNOWLEDGEMENT}

This work was supported by the Soonchunhyang University Research Fund

\section{REFERENCE}

1. Deakin CD, Nolan JP, Soar J, Sunde K, Koster RW, Smith GB, et al. European Resuscitation Council Guidelines for Resuscitation 2010 Section 4. Adult advanced life support. Resuscitation 2010;81(10):1305-52.

2. Wang HE, Balasubramani G, Cook LJ, Lave JR, Yealy DM. Out-of-hospital endotracheal intubation experience and patient outcomes. Ann Emerg Med 2010;55(6):527-37. e6.

3. Do Shin S, Ahn KO, Song KJ, Park CB, Lee EJ. Out-of-hospital airway management and cardiac arrest outcomes: a propensity score matched analysis. Resuscitation 2012;83(3):313-9.

4. Lim JS, Cho YC, Kwon OY, Chung SP, Yu K, Kim SW. Precise minute ventilation delivery using a bag-valve mask and audible feedback. Am J Emerg Med 2012;30(7):1068-71.

5. Kim J, Kim K, Kim T, Rhee JE, Jo YH, Lee JH, et al. The clinical significance of a failed initial intubation attempt during emergency department resuscitation of out-of-hospital cardiac arrest patients. Resuscitation 2014;85(5):623-7.

6. McMullan J, Gerecht R, Bonomo J, Robb R, McNally B, Donnelly J, et al. Airway management and out-of-hospital cardiac arrest outcome in the CARES registry. Resuscitation 2014;85(5):617-22.

7. Deakin CD, Morrison LJ, Morley PT, Callaway CW, Kerber RE, Kronick SL, et al. Advanced life support: 2010 International Consensus on Cardiopulmonary Resuscitation and Emergency Cardiovascular Care Science with Treatment Recommendations. Re- 
suscitation 2010;81(1):e93-e174.

8. Caplan RA, Vistica MF, Posner KL, Cheney FW.. Adverse anesthetic outcomes arising from gas delivery equipment: a closed claims analysis. Anesthesiology1997;87(4):741-8.

9. Deakin C, Peters R, Tomlinson P, Cassidy M. Securing the prehospital airway: a comparison of laryngeal mask insertion and endotracheal intubation by UK paramedics. Emerg Med J 2005;22(1):64-7.

10. 1Frascone RJ, Russi C, Lick C, Conterato M, Wewerka SS, Griffith KR, et al. Comparison of prehospital insertion success rates and time to insertion between standard endotracheal intubation and a supraglottic airway. Resuscitation 2011;82(12):1529-36.

11. Kajino K, Iwami T, Kitamura T, Daya M, Ong ME, Nishiuchi T, et al. Comparison of supraglottic airway versus endotracheal intubation for the pre-hospital treatment of out-of-hospital cardiac arrest. Crit Care 2011;15(5):R236.

12. Duckett J, Fell P, Han K, Kimber C, Taylor C. Introduction of the I-gel supraglottic airway device for prehospital airway management in a UK ambulance service. Emerg Med J 2014;31(6):505-7.

13. Middleton PM, Simpson PM, Thomas RE, Bendall JC. Higher insertion success with the i-gel supraglottic airway in out-ofhospital cardiac arrest: A randomised controlled trial. Resuscitation 2014;85(7):893-7.

14. Je S, Cho Y, Choi HJ, Kang B, Lim T, Kang H. An application of the learning curve-cumulative summation test to evaluate training for endotracheal intubation in emergency medicine. Emerg Med J 2013:291-4.

15. Cummins RO, Chamberlain DA, Abramson NS, Allen M, Baskett PJ, Becker L, et al. Recommended guidelines for uniform reporting of data from out-of-hospital cardiac arrest: the Utstein Style. A statement for health professionals from a task force of the American Heart Association, the European Resuscitation Council, the Heart and Stroke Foundation of Canada, and the Australian Resuscitation Council. Circulation 1991;84(2):960-75.

16. Sakles JC, Chiu S, Mosier J, Walker C, Stolz U. The importance of first pass success when performing orotracheal intubation in the emergency department. Acad Emerg Med 2013;20(1):71-8.

17. SOS-KANTO study group. Cardiopulmonary resuscitation by bystanders with chest compression only (SOS-KANTO): an observational study. Lancet 2007;369(9565):920-6.

18. Yeh ST, Cawley RJ, Aune SE, Angelos MG.. Oxygen requirement during cardiopulmonary resuscitation (CPR) to effect return of spontaneous circulation. Resuscitation 2009;80(8):951-5.

19. Leventis C, Chalkias A, Sampanis MA, Foulidou X, Xanthos T. Emergency airway management by paramedics: comparison between standard endotracheal intubation, laryngeal mask airway, and I-gel. Eur J Emerg Med 2014;21(5):371-3.

20. Lee DW, Kang MJ, Kim YH, Lee JH, Cho KW, Kim YW, et al. Performance of intubation with 4 different airway devices by unskilled rescuers: manikin study. Am J Emerg Med 2015;33(5):691-6.

21. Ruetzler K, Gruber C, Nabecker S, Wohlfarth P, Priemayr A, Frass M, et al. Hands-off time during insertion of six airway devices during cardiopulmonary resuscitation: a randomised manikin trial. Resuscitation 2011;82(8):1060-3.

22. Gatward JJ, Thomas MJ, Nolan JP, Cook TM. Effect of chest compressions on the time taken to insert airway devices in a manikin. Br J Anaesth 2008;100(3):351-6. 